\title{
Blocking Glutamate-Mediated Inferior Olivary Signals Abolishes Expression of Conditioned Eyeblinks But Does Not Prevent Their Acquisition
}

\author{
Andrew J. Carrel, Gary D. Zenitsky, and Vlastislav Bracha \\ Department of Biomedical Sciences, College of Veterinary Medicine, Iowa State University, Ames, Iowa 50011
}

The inferior olive (IO) is considered a crucial component of the eyeblink conditioning network. The cerebellar learning hypothesis proposes that the IO provides the cerebellum with a teaching signal that is required for the acquisition and maintenance of conditioned eyeblinks. Supporting this concept, previous experiments showed that lesions or inactivation of the IO blocked CR acquisition. However, these studies were not conclusive. The drawback of the methods used by those studies is that they not only blocked task-related signals, but also completely shut down the spontaneous activity within the IO, which affects the rest of the eyeblink circuits in a nonspecific manner. We hypothesized that more selective blocking of task-related IO signals could be achieved by using injections of glutamate antagonists, which reduce, but do not eliminate, the spontaneous activity in the IO. We expected that if glutamate-mediated IO signals are required for learning, then blocking these signals during training sessions should prevent conditioned response (CR) acquisition. To test this prediction, rabbits were trained to acquire conditioned eyeblinks to a mild vibrissal airpuff as the conditioned stimulus while injections of the glutamate antagonist $\gamma$-D-glutamylglycine were administered to the IO. Remarkably, even though this treatment suppressed CRs during training sessions, the postacquisition retention test revealed that CR acquisition had not been abolished. The ability to acquire CRs with IO unconditioned stimulus signals that were blocked or severely suppressed suggests that mechanisms responsible for CR acquisition are extremely resilient and probably less dependent on IO-task-related signals than previously thought.

\section{Introduction}

In delay classical conditioning, a biologically neutral conditioned stimulus (CS) is followed by an aversive unconditioned stimulus (US), the timing of which overlaps and coterminates with the CS. Animals learn to respond to the CS with conditioned responses (CRs). The cerebellar learning hypothesis proposes that the inferior olive (IO) supplies the cerebellum with the US "teaching" signal deemed essential for learning and maintaining CRs (Raymond et al., 1996; De Zeeuw and Yeo, 2005; Jirenhed et al., 2007). This hypothesis is consistent with studies showing IO neurons firing in response to periocular stimulation (Gellman et al., 1983; Weiss et al., 1993) and studies preventing CR acquisition in rabbits by either permanently lesioning the IO or inactivating it using lidocaine (McCormick et al., 1985; Yeo et al., 1986; Mintz et al., 1994; Welsh and Harvey, 1998).

However, these CR acquisition studies could not avoid cerebellar tonic malfunction, which is known to be triggered by IO lesions or inactivation (Colin et al., 1980; Montarolo et al., 1982; Batini et al., 1985). Therefore, it is unknown whether blocked IO

Received July 2, 2012; revised April 1, 2013; accepted April 16, 2013.

Author contributions: A.J.C., G.D.Z., and V.B. designed research; A.J.C., G.D.Z., and V.B. performed research; A.J.C., G.D.Z., and V.B. analyzed data; A.J.C., G.D.Z., and V.B. wrote the paper.

This work was supported by the National Institutes of Health (Grant R01 NS036210).

The authors declare no competing financial interests.

Correspondence should be addressed to Vlastislav Bracha, Department of Biomedical Sciences, College of Veterinary Medicine, 2032 Vet Med, lowa State University, Ames, IA 50011. E-mail: vbracha@iastate.edu.

DOI:10.1523/JNEUROSCI.3129-12.2013

Copyright $\odot 2013$ the authors $\quad 0270-6474 / 13 / 339097-07 \$ 15.00 / 0$ sensory signaling or a dysfunctional cerebellum prevented learning. Ideally, to test the role of IO US signals in CR acquisition, one must block these signals without affecting IO and cerebellar spontaneous activity.

A potentially more selective method for blocking IO task-related signals is suppressing their US inputs by glutamate antagonists, which have been shown to block responses to trigeminal stimulation (Lang, 2001) and to reduce, but not abolish, the IO's spontaneous firing rate (Lang, 2001). Even this relatively modest reduction of IO spontaneous firing has a major nonspecific effect on cerebellar output neurons - it silences them (Zbarska et al., 2007, 2008). Clearly, successfully testing the IO's teaching role will require methods that block IO US signals while compensating for tonic changes in the cerebellum. We hypothesized that this issue could be resolved by using combined drug injection protocols (Zbarska and Bracha, 2012) to block glutamatergic signals while concomitantly increasing IO spontaneous activity during CR acquisition.

In the first part of this study, we examined the effects of infusing the glutamate antagonist $\gamma$-D-glutamylglycine (DGG) into the IO on CR acquisition without compensating for its effects on the spontaneous IO activity. Rabbits were initially trained using a tone CS. After tone training, DGG was administered to the contralateral dorsal accessory olive to determine the optimal location and dose needed for blocking glutamate neurotransmission in the eyeblink representation area (Weiss et al., 1993), as indexed by complete CR abolition (Zbarska et al., 2007). Rabbits were then trained using a weak airpuff to the vibrissal pad (vCS) while blocking IO glutamate during each training session. 
We hypothesized that blocking glutamate neurotransmission in the IO would prevent new CR acquisition due to the IO US signals being blocked and also due to the resultant suppression of anterior cerebellar interposed nucleus (IN) activity (Zbarska et al., 2007, 2008), which when induced by direct IN inactivation blocks learning (Krupa et al., 1993). Here we report that, contrary to expectations, IO injections of DGG that reliably abolished previously learned tone-evoked CRs did not prevent acquisition of vCS-evoked CRs. These findings indicate that the CR acquisition mechanism is extremely resilient and can function in the presence of blocked or severely diminished IO US signals and on the background of CR-abolishing cerebellar tonic malfunction.

\section{Materials and Methods}

\section{Subjects}

Experiments were performed on 12 male New Zealand white rabbits (Harlan Laboratories) that weighed $2.5-3.0 \mathrm{~kg}$ and were 3-4 months old at the time of surgery. Rabbits were housed individually on a $12 \mathrm{~h} \mathrm{light/}$ dark cycle and provided food and water ad libitum. All experiments were performed in accordance with the National Institutes of Health's "Principles of Laboratory Animal Care" (publication no. 86-23, revised 1985), the American Physiological Society's "Guiding Principles in the Care and Use of Animals," and the protocol approved by Iowa State University's Animal Care and Use Committee.

\section{Surgery}

Surgical implantations were performed using aseptic techniques on naive rabbits anesthetized with a mixture of ketamine $(50 \mathrm{mg} / \mathrm{kg})$, xylazine $(6$ $\mathrm{mg} / \mathrm{kg})$, and acepromazine $(1.5 \mathrm{mg} / \mathrm{kg})$. The head was secured in a stereotaxic apparatus with lambda positioned $1.5 \mathrm{~mm}$ ventral to bregma. After exposing the skull and affixing three stainless steel anchor screws, a stainless steel injection guide tube (27-gauge thin-wall) was implanted stereotaxically targeting the dorsal aspects of the right IO using the equation $(0.69 \mathrm{X}+4.5)-\mathrm{X}$ rostral from lambda, where $\mathrm{X}$ is the horizontal distance between bregma and lambda in millimeters; $1.0 \mathrm{~mm}$ lateral; and $23.4 \mathrm{~mm}$ ventral from lambda. To protect its patency, a 33-gauge stainless steel stylet was inserted in the guide tube between experiments. The guide tube, anchor screws, and a small plastic block designed to accommodate an airpuff delivery nozzle and eyeblink sensor were secured in place with dental acrylic. All animals were treated with antibiotics for $5 \mathrm{~d}$ while recovering from surgery.

\section{Training procedures}

Before surgery, rabbits were adapted to a restraint box (Plas-Labs) inside a sound-attenuating chamber for $30 \mathrm{~min}$ on two consecutive days. After recovery from surgery, rabbits were given one additional day of box adaptation. Box-adapted rabbits were assigned to either the experimental or control group and conditioned using the delay classical conditioning paradigm until they reached $\geq 90 \%$ CRs for three consecutive training days. The initial CS was a $450 \mathrm{~ms}, 85 \mathrm{~dB}, 1 \mathrm{kHz}$ tone superimposed on a $70 \mathrm{~dB}$ white noise background. The tone CS coterminated with a $100 \mathrm{~ms}$, $36 \mathrm{psi}$ (at the source) US directed at the left cornea. The interstimulus interval was $350 \mathrm{~ms}$ and the intertrial interval varied pseudorandomly between 15 and 25 s. Each training session consisted of 100 paired trials per day.

\section{Injection procedures and acquisition experiments}

Intracranial microinjections were delivered using a 33-gauge stainless steel injection needle that was connected to a $10 \mu$ l Hamilton syringe via transparent Tygon tubing. The AMPA/kainate and NMDA receptor antagonist, DGG (Tocris Bioscience) was dissolved in aCSF and its $\mathrm{pH}$ was adjusted to $7.4 \pm 0.1$. The injections were performed manually at a rate of $0.25 \mu \mathrm{l} / \mathrm{min}$.

Determining IO injection sites. In the first part of the study, rabbits were subjected to functional mapping sessions in which they were injected with DGG $(100 \mathrm{nmol} / \mu \mathrm{l})$ to determine the optimal location and amount of DGG required to quickly abolish previously learned tone-evoked CRs. The injection needle was inserted in the guide tube before starting the experiment. Rabbits were presented with 40 preinjection trials to ensure that there were no effects on CR performance due to needle insertion. After the drug injection, an additional 160 postinjection trials were presented to the rabbit. In the first mapping experiment, DGG was injected at a depth that corresponded to the tip of the injection guide tube. Subsequent DGG injections were given every 20 trials until we observed a drug effect or a maximum of $2 \mu \mathrm{l}$ had been injected. If no drug effect on CR incidence was observed, the injection needle was advanced $0.5 \mathrm{~mm}$ deeper the following day and the mapping session was repeated. This procedure was performed until DGG injections completely abolished CRs or until the needle had reached the base of the skull.

$v C S$ acquisition training. After determining the effective injection site for DGG to abolish tone-evoked CRs, the main part of the study commenced. This began with calibration of the 450 ms weak airpuff CS directed at rows B and C of the left mystacial vibrissae (vCS) that would be used for the acquisition protocol. The strength of this CS was individually calibrated for each rabbit (2-20 psi) to an intensity that was just below the threshold for eliciting an $\alpha$ response (for a more detailed description, see Carrel et al., 2012). Once the intensity of the vCS was determined, the acquisition experiment began. This part of the study entailed $3 \mathrm{~d}$ of acquisition with 100 paired vCS-US trials per day. Before each vCS acquisition training session started, the injection needle was inserted first, then 10 tone CS-US presentations were delivered to test for possible needle insertion effects. The experimental group received IO DGG injections before each vCS acquisition session and the control group received IO vehicle injections. To ensure a reliable blockade of IO glutamate receptors, rabbits were injected with double the volume of DGG that was needed to abolish CRs in the mapping experiment. This same volume was administered in vehicle injections. After a waiting period equivalent to the previously established latency for DGG-induced CR abolition, paired tone CS-US trials were resumed to confirm the drug effect by observing DGG-induced abolition of previously learned toneevoked CRs. During these postinjection tone trials, CRs were considered abolished when no more than one CR per 10-trial block was present. In rare instances, an additional DGG supplement was given to achieve complete abolition of CRs. Once CRs were abolished, acquisition training using the vCS began. The vCS acquisition sessions were divided into blocks of 10 trials consisting of nine vCS-US trials and one tone CS-US trial. The probe tone CS trials during training were used to monitor the continuing DGG effect by observing the performance of previously learned CRs. These procedures were repeated in all vCS training sessions.

Retention test and additional training with no drugs injected. After $3 \mathrm{~d}$ of vCS acquisition, rabbits were given $1 \mathrm{~d}$ of rest to recover from the DGG effects, after which time they were given a retention test. The retention test consisted of $40 \mathrm{vCS}$-alone trials to assess whether any learning occurred during the previous training under the influence of IO DGG. Immediately after the retention test, rabbits were trained in daily sessions of 100 paired vCS-US trials until they reached an asymptotic level of responding for $3 \mathrm{~d}$.

\section{Data recording and analysis}

Movements of the eyelids were recorded using a wide field-of-view infrared sensor that measures the amount of infrared light reflected from the eye and periorbital region (Ryan et al., 2006). The output of the sensor was amplified, digitized ( $25 \mathrm{kHz}, 12$-bit A/D converter), and stored using a custom-made data acquisition system. An infrared video system installed in the experiment chamber was used to monitor the behavior of the rabbits and for positioning the infrared sensor.

Eyeblink data were acquired starting $250 \mathrm{~ms}$ before onset of the CS and ending $800 \mathrm{~ms}$ beyond the US onset for a total of $1400 \mathrm{~ms}$ per trial. Eyeblink responses were analyzed offline for the presence of $\alpha$ responses, CRs, and URs, each defined by their onset latencies. An $\alpha$ response was classified as any response up to $80 \mathrm{~ms}$ after CS onset, a CR as an eyelid movement occurring from $81 \mathrm{~ms}$ after CS onset up to the US onset, and a UR as any response after the US onset. Trials containing spontaneous eyeblinks before CS onset were removed from further analyses. The threshold for eyelid movements was set to 5 SDs of the baseline signal noise, which corresponded to an approximately $0.15 \mathrm{~mm}$ decrease in eyelid aperture. Mean CR incidence and latency were calculated for con- 

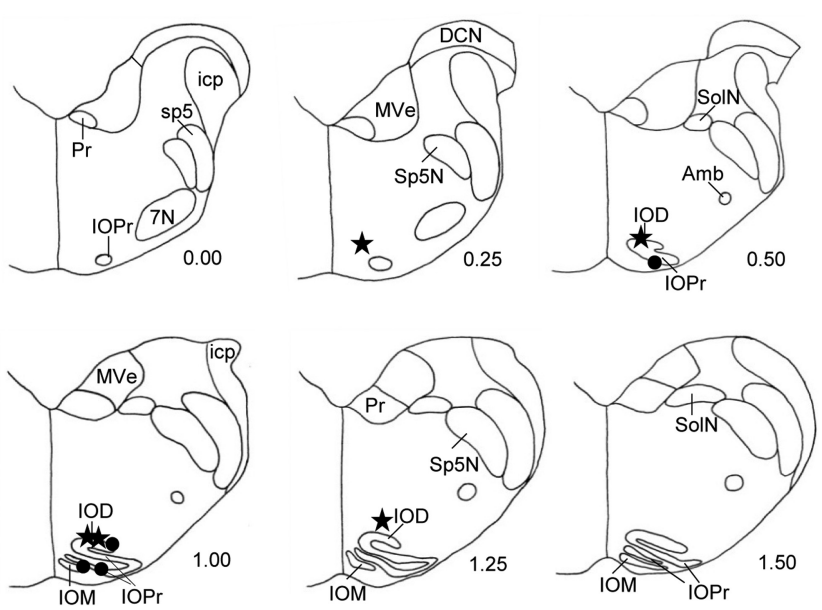

Figure 1. Locations of injection sites in the 10 for the experimental group (stars) and the control animals (circles). The injection sites for individual animals were plotted on a set of standardized coronal sections of the rabbit medulla arranged in rostral-to-caudal order with the upper left section being the most rostral. The numbers on the lower right side of each section represent the anterior-posterior distance in millimeters from the rostral part of the dorsal accessory 10. All injection sites were located in or adjacent to the rostral portion of the 10 complex. IOD indicates dorsal accessory 10; 10M, medial I0; IOPr, principal I0; Sp5N, spinal trigeminal nucleus; sp5, spinal trigeminal tract; Pr, prepositus hypoglossal nucleus; $7 \mathrm{~N}$, facial nucleus; icp, inferior cerebellar peduncle; MVe, medial vestibular nucleus; SoIN, solitary nucleus; Amb, ambiguous nucleus; DCN, dorsal cochlear nucleus.

secutive blocks of 10 trials. The data were pooled from individual rabbits and statistically analyzed using repeated-measures ANOVA followed by individual and simultaneous contrast analyses. All group data are reported as means \pm SEM, with an $p=0.05$ declaring significance.

\section{Histology}

After all experiments were completed, rabbits were deeply anesthetized with a mixture of ketamine $(100 \mathrm{mg} / \mathrm{kg})$, xylazine $(12 \mathrm{mg} / \mathrm{kg})$, and acepromazine $(3 \mathrm{mg} / \mathrm{kg})$. Injection sites were marked by injecting $0.75-$ $1.0 \mu \mathrm{l}$ of tissue-marking dye. Rabbits were transcardially perfused with $1 \mathrm{~L}$ of PBS followed by $1 \mathrm{~L}$ of tissue fixative (10\% neutral-buffered formalin). Excised brains were stored in a solution of $30 \%$ sucrose and $10 \%$ formalin. Brains were sectioned coronally in $50 \mu \mathrm{m}$ slices on a freezing microtome. Subsequently, sections were mounted on gelatin-coated slides, dried, and stained with Luxol blue and neutral red. Injection site locations were identified using bright light microscopy and plotted on standardized sections of the rabbit medulla.

\section{Results}

Overall, blocking IO fast glutamate receptors while training rabbits to a vCS did not block learning as demonstrated by the postacquisition retention test. This was surprising because, during the acquisition sessions, CR expression was abolished. In agreement with this result, rabbits showed a near asymptotic level of vCSevoked CRs on day 1 of postacquisition training.

A total of 12 rabbits were included in the acquisition experiments. One rabbit was excluded from the study due to consistent URs to the lowest intensities of the vCS. Another rabbit was killed due to poor health. After histological analysis, one additional subject was excluded from the results due to a misplaced implant. Therefore, five animals were included in the experimental group and four in the control group. The histological reconstruction of IO injection sites for the remaining nine animals revealed that all subjects had their injections administered either directly in or proximal to the rostral part of the dorsal accessory IO (Fig. 1).

The IO mapping tests: effects of DGG on tone CS-evoked CRs Determining the optimal depth for IO injections and the dose of DGG required for blocking IO signals in the eyeblink-related area
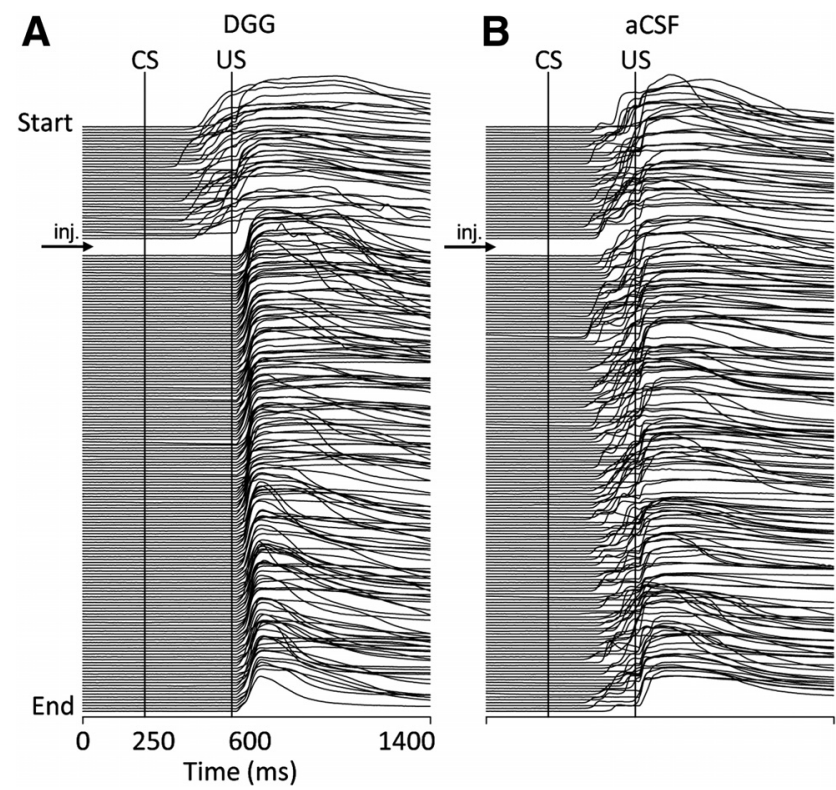

Figure 2. Individual examples from the same animal showing the effects of injecting DGG or vehicle in the 10 on expression of tone CS-evoked CRs in the 10 mapping part of the study. Each experiment starts at the top and each blink represents one trial of the 200-trial experiment. Upward deflections of the trace between the vertical CS and US markers denote conditioned eyeblinks. The timing of injections is shown by an arrow in each stack plot. $A$, Effect of DGG injected in the 10 after 40 preinjection trials. DGG immediately abolished CRs and this effect lasted for the remainder of the experiment. $\boldsymbol{B}$, Control experiment in which aCSF was injected in the I0. The vehicle had no effect on CRs.

of the IO was conducted in rabbits pretrained with the tone CS. The suppression of tone CS-evoked CRs served as a functional index of an adequate DGG injection. Before the DGG injection, all rabbits exhibited well timed, large-amplitude CRs to the tone CS (Fig. 2A). After DGG administration at effective sites, CR performance quickly deteriorated and CRs were abolished for the remainder of the experiment (Fig. 2A, Fig. 3). The volume required for maintenance of DGG-induced abolition for the 160 postinjection trials ranged from 0.5 to $1.5 \mu$ l. This suppression of $\mathrm{CR}$ incidence was significant when compared with the preinjection performance $\left(F_{(1,14)}=1574.2, p<0.0001\right)$ or to control injections of vehicle $\left(F_{(1,14)}=406.3, p<0.0001\right)$. The effect of DGG on CR incidence in the control and experimental groups was similar $\left(\left(F_{(1,14)}=0.96, p=0.34\right.\right.$; Fig. 3). These data demonstrated that, at selected injection sites, DGG reached eyeblink-related areas in the IO and that blocking glutamate neurotransmission was sufficient to abolish previously learned CRs for the duration of the upcoming CR acquisition experiment.

\section{CR acquisition while blocking glutamatergic neurotransmission in the IO}

After finding the optimal IO injection location and dose of DGG required to abolish previously learned CRs, the acquisition experiment was conducted. Rabbits in the experimental group received a dose of DGG that was double the volume needed to abolish CRs during the IO mapping phase of the experiment. This drug excess was used to increase the likelihood that all glutamate signaling in the eyeblink-related portions of the IO was indeed blocked. During the acquisition phase of the study, rabbits were injected with either DGG or vehicle before each training session and trained to the vCS for $3 \mathrm{~d}$. The DGG treatment prevented expression of CRs to both CS modalities. Figure $4 A, C$ shows individual examples of eyeblink responses to the vCS on day 3 of 


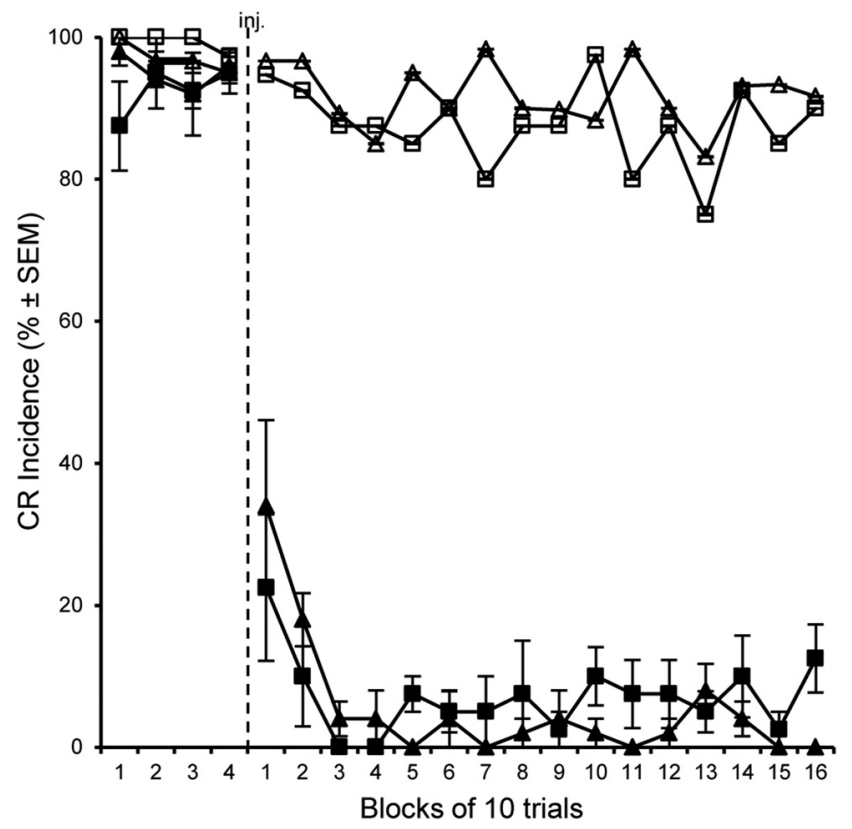

Figure 3. Group data for tone CS-evoked CR incidence ( \pm SEM) after DGG or vehicle injections in the I0 for the experimental $(n=5)$ and control $(n=4)$ groups. The dashed vertical line indicates the time of injection. The abscissa represents blocks of 10 trials, which are numbered separately for the preinjection and postinjection periods. DGG injections in both the experimental group (black triangles) and control group (black squares) rapidly abolished CRs and CR expression did not recover until the end of the experiment. Injections of vehicle had no effect on CR incidence.

acquisition, contrasting the effects of DGG and aCSF. In addition to a few small-amplitude spontaneous eyeblinks, the rabbit in the experimental group exhibited no CRs to the vCS (Fig. 4A). Conversely, the control rabbit had a high number of large and well timed vCS-evoked CRs on the same day of training (Fig. 4C). In the tone CS probe trials of experimental animals, tone-evoked CRs were completely abolished (Fig. $4 B$ ), indicating a major and lasting DGG effect during the entire acquisition experiment. Conversely, control rabbits consistently exhibited normally timed, tone-evoked CRs (Fig. 4D). The consistent effect of DGG on vCS-related CR expression is shown in Figure 5A. Over the course of all three acquisition sessions, the frequency of blinks in the CS-US interval in this rabbit did not exceed the spontaneous eyeblink rate and the small spontaneous blinks were intermixed with occasional slight eyelid-opening movements (e.g., several of the last trials on day 3; Fig. 5). Overall, control rabbits exhibited rapid CR acquisition culminating with an asymptotic CR performance by day 3 (Fig. 4, Fig. 6). Rabbits trained while being injected with DGG showed virtually no vCS-evoked CRs on all $3 \mathrm{~d}$ (Fig. 5, Fig. 6). This level of responding was significantly lower from the first day of training $\left(F_{(1,7)}=22.5, p=0.002\right)$ compared with the control group's rapid rise to asymptotic performance.

\section{Retention test}

After acquisition, a no-injection, 40-trial, vCS-alone retention test was performed. In this test, the experimental group exhibited a surprisingly high incidence of vCS-evoked CRs (35\%), providing unequivocal evidence that learning occurred during the DGG phase of acquisition training (Fig. 5B, Fig. 6A). An individual example of CR performance in the retention test for a rabbit in the experimental group is shown in Figure $5 B$. This animal exhibited a high level of well timed CRs to the vCS throughout the retention test and also showed signs of extinction toward the end of the session. The average onset latency of CRs in the retention test was $202.3 \pm 12.9 \mathrm{~ms}$, which was significantly later than the $42.1 \pm 1.6 \mathrm{~ms}$ vibrissal UR onset observed during CS intensity calibration $\left(t_{12}=9.06, p<0.001\right)$. One of the five experimental rabbits was extremely sensitive to the vCS; therefore, during acquisition training, we had to select the lowest vCS intensity available in our airpuff delivery system to prevent vCS-evoked URs. This rabbit failed to show high CR incidence in the retention test, but exhibited above-normal CR incidence during each day of postacquisition training. Therefore, although rabbits in the control group exhibited a higher mean level of CRs during retention, individual performances varied such that the two groups were not statistically different $\left(F_{(1,7)}=4.38, p=0.075\right)$.

\section{Postacquisition training}

During the first day of postacquisition training, both groups of rabbits exhibited high CR incidence, and the asymptotic level of performance was maintained through all three training sessions (Fig. 6). Consistent with learning having occurred during the DGG acquisition phase, the learning curve of the experimental group was indistinguishable from controls ( $g r o u p F_{(1,7)}=0.036$, $p=0.85$ and group ${ }^{*}$ day $F_{(2,14)}=0.81, p=0.46$; Fig. $\left.6 A\right)$.

\section{Discussion}

This study tested whether interfering with US signals by blocking glutamate neurotransmission in the IO affects CR acquisition to a vCS. As expected, DGG prevented CR expression to both the vCS and previously learned tone CS (Fig. 3, Fig. 5, Fig. 6). Despite being unable to generate CRs to either CS modality during acquisition, rabbits exhibited vCS-evoked CRs in the injection-free retention test. Therefore, rabbits acquired CRs even though IO US signals to the cerebellum were suppressed.

Prevailing concepts of eyeblink conditioning postulate that the IO provides the cerebellum with a US teaching signal that is required for CR acquisition and maintenance (Thompson, 1986; De Zeeuw and Yeo, 2005). Electrophysiological studies have demonstrated that the dorsal accessory olive responds to somatosensory stimulation of the periocular region (Gellman et al., 1983; Weiss et al., 1993). Further confirmation of the IOdependent cerebellar learning hypothesis required showing that blocking IO US signals prevented CR acquisition and caused CR extinction. Although the effect on acquisition has been demonstrated previously (McCormick et al., 1985; Yeo et al., 1986; Welsh and Harvey, 1998), results of studies blocking IO signals in trained animals were less conclusive. Although Yeo et al. (1986) found that IO lesions immediately abolished previously learned CRs, McCormick et al. (1985) reported that IO lesions produced gradual, extinction-like CR suppression. A similarly delayed extinction effect resulted from reversibly blocking glutamate-mediated IO US signals in trained rabbits (Medina et al., 2002).

Our recent electrophysiological examination of blockingtask-related IO signals in trained rabbits casted serious doubts on the approach used in these previous studies. We found that blocking IO signals with microinjections of muscimol (a GABA agonist) or DGG or NBQX (fast glutamate receptor antagonists) invariably abolished CRs (Zbarska et al., 2007, 2008). In these studies, our single-unit recordings of neuronal activity in deep cerebellar nuclei unveiled the neurophysiological mechanism of CR abolition. Like other studies of tonic effects of IO lesions (Colin et al., 1980; Montarolo et al., 1982; Batini et al., 1985), we found that these treatments suppressed neuronal activity in cerebellar interposed nuclei. Because it was known that incomplete 
A

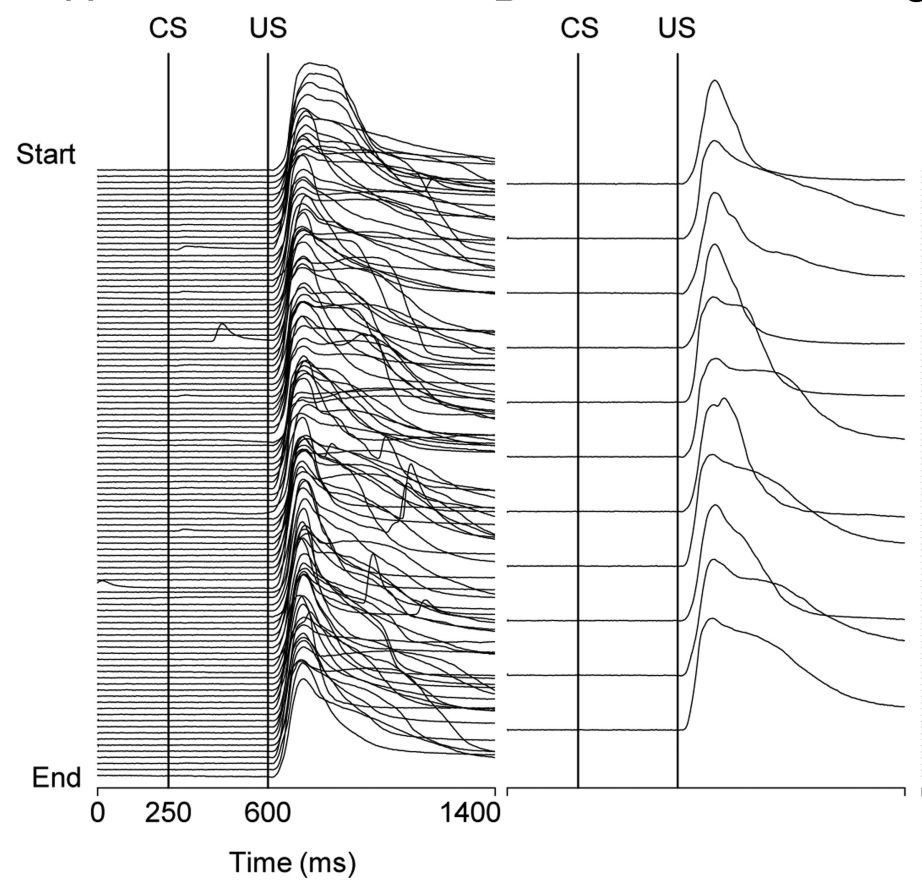

C

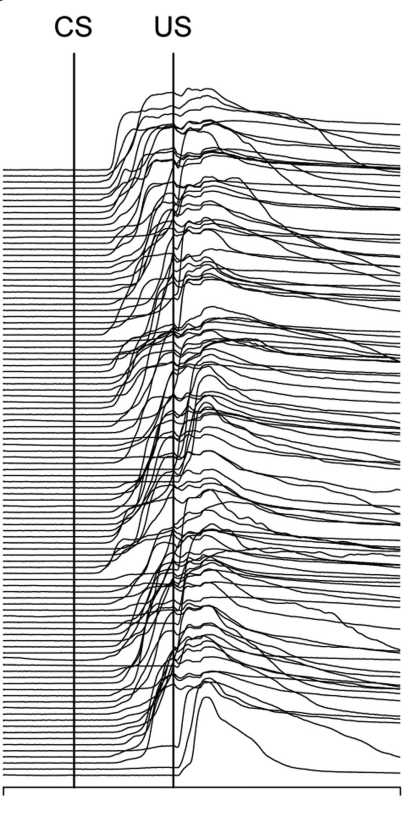

D

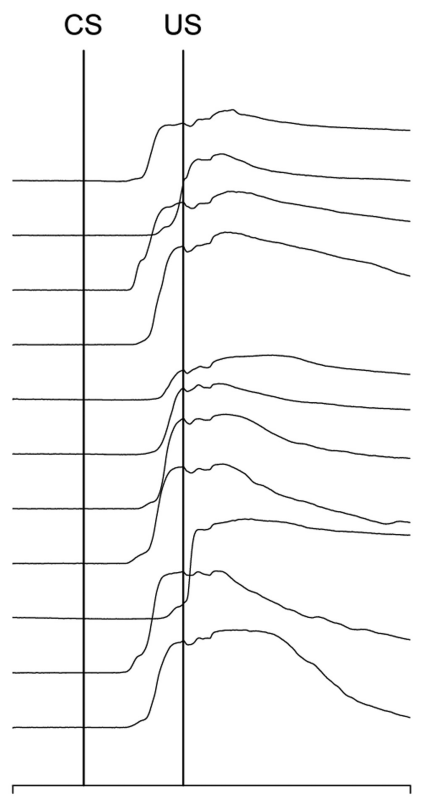

Figure 4. Individual examples of vibrissal and tone CS trials from day 3 of acquisition with DGG or vehicle injections in the I0. $A$, Example showing a 100 -trial vCS acquisition experiment after a DGG injection. The rabbit was exhibiting no CRs on this last day of acquisition. $\boldsymbol{B}$, The probe tone CS trials delivered to the rabbit during the same acquisition session as in $A$. The DGG injection prevented expression of previously learned CRs. C, Stack plot of eyeblinks of a control animal that was injected with vehicle. At this stage of acquisition, the control rabbit expressed well developed CRs. D, Eyeblinks in the tone CS trials presented in the same session as in $\boldsymbol{C}$. In these trials, the control animal showed well timed CRs to the previously learned tone CS.

A

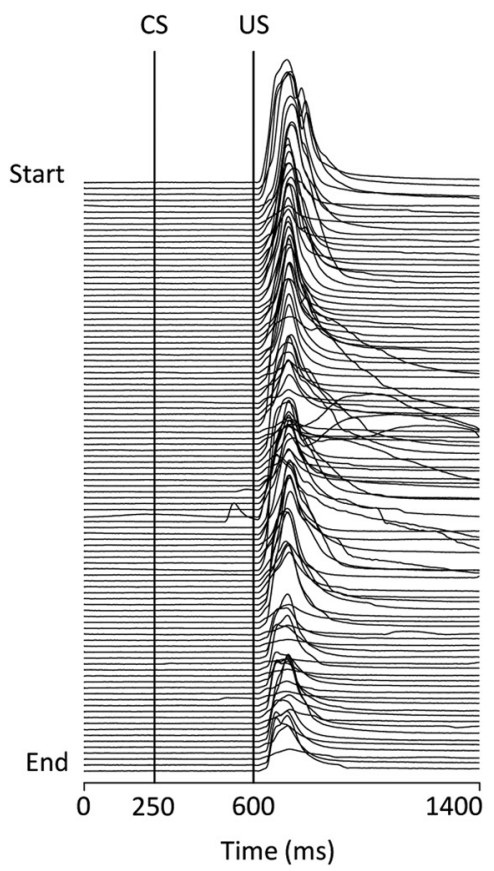

Day 2

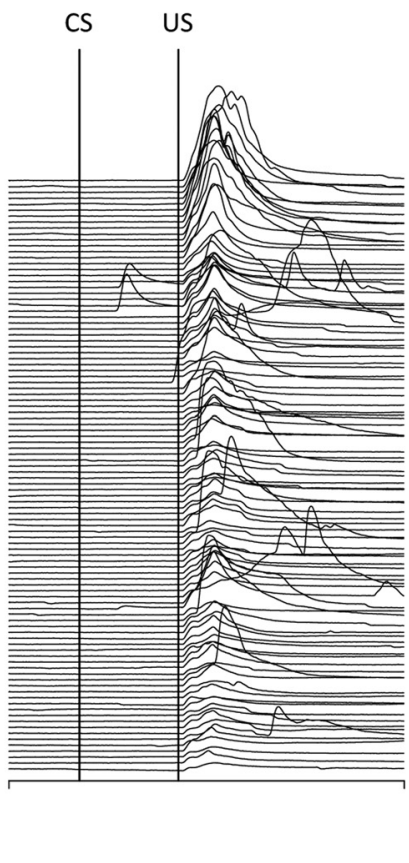

Day 3

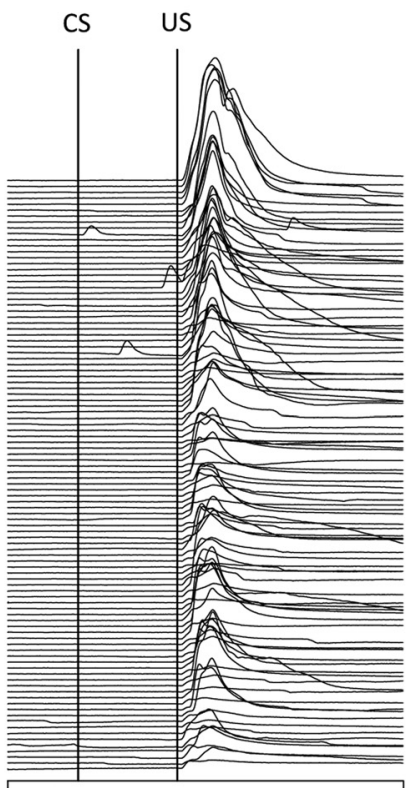

B

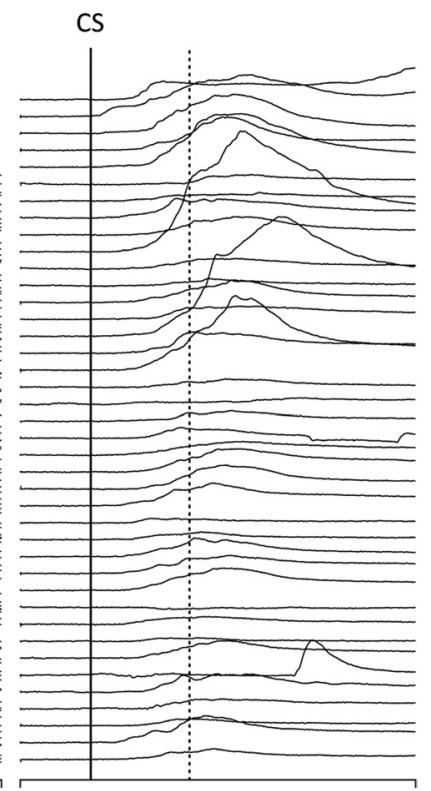

Figure 5. A complete printout of all eyeblinks generated by one DGG-injected experimental rabbit during 3 days of vCS training and in the retention test. $A$, With the exception of several spontaneous responses, DGG in this rabbit suppressed the expression of CRs throughout the acquisition phase of the study. $\boldsymbol{B}$, Despite showing no CRs during acquisition, the same animal exhibited well formed vCS-evoked CRs in the vCS-alone retention test. Dashed vertical line shows where the onset of the US would normally occur in a paired stimuli experiment.

suppression of IN activity by direct activation of IN GABA $\mathrm{A}_{\mathrm{A}}$ receptors suppresses CR and UR amplitudes (Jiménez-Díaz et al., 2004), and that more extensive suppression of cerebellar nuclear activity blocks CR expression (Welsh and Harvey, 1991; Bracha et al., 1994), we concluded that IO-manipulation-induced suppression of cerebellar nuclear activity caused the CR abolition directly. Because of their side effects (i.e., nonspecific suppression of cerebellar nuclear activity), IO lesions and reversibly blocking 
A
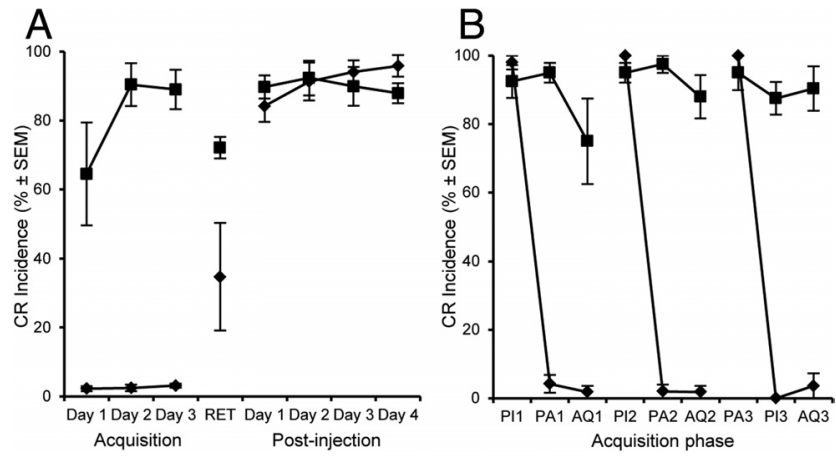

Figure 6. Group effects on vCS-evoked CR incidence ( \pm SEM) during acquisition, retention test (RET), and postacquisition training for the experimental group (diamonds, $n=5$ ) and control group (squares, $n=4$ ). $A$, DGG injections suppressed expression of any vCS-evoked CRs during acquisition, but did not block learning, as shown by the presence of relatively high CR incidence in the retention test. The control group acquired CRs to the vCS quickly and CR expression reached asymptote by day 2. Both groups showed asymptotic levels of CR incidence from the first day of the no-injection, postacquisition training. $\boldsymbol{B}$, Tone CR incidence during the $3 \mathrm{~d}$ of acquisition. Both groups showed high levels of CRs during the preinjection (PI) trials. DGG injections in the experimental group blocked expression of tone-evoked CRs in both the preacquisition $(\mathrm{PA})$ and acquisition $(\mathrm{AQ})$ trials. Vehicle injections in the control group had no effect on expression of tone-evoked CRs.

IO neurotransmission are invalid methods for testing the functional significance of IO signals in eyeblink conditioning.

Investigating the IO's contribution to eyeblink conditioning requires new approaches that not only block IO US signals, but also minimize disruption of tonic activity of cerebellar circuits (for review, see Bracha et al., 2009). A pioneering approach toward this end was developed by Bengtsson et al. (2007), who suppressed IO signals for short periods of time by stimulating the cerebellar-olivary pathway before each US application. Although well suited for acute experiments in decerebrate ferrets, implementing this technically complex approach in awake animals acquiring CRs would be exceedingly difficult. As an alternative, we developed combined injection protocols to block IO-task-related signals while maintaining near-normal cerebellar activity (Zbarska and Bracha, 2012). Here we report the baseline effect of blocking glutamate-mediated IO signals during acquisition without compensating for associated shifts in cerebellar spontaneous activity. We hypothesized that rabbits trained in this way would not acquire CRs because DGG renders cerebellar circuits dysfunctional and/or blocks the IO US signals presumably required for learning. Surprisingly, we found that injections of DGG did not prevent new $\mathrm{CR}$ acquisition. The simplest explanation for this observation is that glutamate-mediated IO US signals perhaps contribute to, but are not required for, CR acquisition. This contradicts a major tenet of the cerebellar learning hypothesis, which states that IO US signals are pivotal for learning, and suggests instead extracerebellar substrates of learning. Our finding does not, however, preclude cerebellar learning in general. Even though DGG-injected animals showed clear evidence of learning in the retention test, their CR incidence was lower than in control animals. Therefore, it is possible that, in normal conditions, the IO US signals are not necessary, but can enhance CR acquisition. Although IO US signals might not be essential, other evidence suggests that the intermediate cerebellum indeed plays a role in acquisition (Bracha et al., 1998; Kellett et al., 2010). If this is the case, then the underlying mechanism would have to be remarkably resilient to withstand IO DGG-induced shifts in the spontaneous activity of the cerebellar cortex and nuclei and would have to rely on mossy fibers for both CS and US information.
If the $\mathrm{IO}$ is not required for $\mathrm{CR}$ acquisition, then why did IO lesions (McCormick et al., 1985; Yeo et al., 1986) and inactivation with lidocaine (Welsh and Harvey, 1998) prevent learning? Olivary neurons are spontaneously active at a rate of $1-2 \mathrm{~Hz}$ (Lang, 2001). These two interventions completely silence the IO, including spontaneous climbing fiber activity, thereby generating nonspecific effects on cerebellar physiology that are more disruptive than selectively blocking IO glutamate, which is less intrusive. Even though the latter method blocks the IO's responses to sensory inputs, it reduces, but does not eliminate, spontaneous firing (Lang, 2001). The DGG-induced decrease in IO activity was sufficient to suppress IN activity via tonic interactions to levels precluding CR expression. However, by preserving residual IO spontaneous activity, DGG's effect was not severe enough to shift the activity in cerebellar eyeblink-related areas beyond the physiological range required for learning.

Before rejecting an essential role for the IO in CR acquisition, alternate interpretations should be considered. If injections did not eliminate all IO US signals, then the residual US-evoked IO activity may have sufficed to support acquisition. However, this scenario seems unlikely. We incorporated multiple functional tests of injection effectiveness to reduce the likelihood of an incomplete block of US signals. Specifically, to ensure drug infusion into locations relevant to the eyeblink, we selected only sites where small DGG injections abolished previously learned, tone CS-evoked CRs (Zbarska et al., 2007). Then, to ensure a complete and lasting drug effect during training, we doubled the DGG dose required for $\mathrm{CR}$ abolition and verified its effect on tone CSevoked CRs before initiating each acquisition session. Moreover, throughout each acquisition session, we confirmed the lasting drug effect using recurring tone-CS probe trials to verify $\mathrm{CR}$ abolition. All of these precautions confirmed that injections were administered into the eyeblink area of the IO and that the resulting glutamate block was extensive enough to render rabbits incapable of generating both previously learned and newly acquired CRs. Finally, we histologically verified that injection sites were centered on the rostral part of the dorsal accessory olive, which contains the contralateral eyeblink representation (Weiss et al., 1993). However, these functional tests only indirectly measure the extent that DGG blocked US signals. It is possible that CR expression is more vulnerable to DGG-induced shifts of tonic cerebellar activity than is acquisition. Therefore, we cannot exclude that a dysfunctional cerebellum was still receiving residual IO US signals sufficient to support learning. Even though this seems unlikely, future electrophysiological experiments are required to test this interpretation.

Another factor enabling rabbits to acquire CRs in the present study could be the CS modality. We used a weak vibrissal airpuff because it produces acquisition of cerebellum-dependent CRs faster than traditional CS modalities such as a tone (Carrel et al., 2012). Therefore, by reducing the number of training sessions, the use of the vCS minimizes the likelihood of a failed drug injection during multiple drug administrations. This feature of vCS conditioning is highly advantageous in acquisition experiments in which one compromised injection that fails to block CRs could invalidate the experiment. On the downside, we cannot exclude that learning a nontraditional CS-sensory modality is differentially dependent on IO signals. The CS signal, whether auditory or trigeminal, is sent to the lateral pontine nuclei and then to the eyeblink-related areas of the cerebellum (Halverson and Freeman, 2010). However, the sensory trigeminal nuclei also send direct projections to the cerebellum (Van Ham and Yeo, 1992), which could contribute to faster acquisition and also to the ability 
to acquire CRs while blocking IO inputs. This possibility could be tested by blocking IO glutamate during tone-CS conditioning. An additional noteworthy finding comes from one rabbit that did not learn during the acquisition protocol. Histological placement of his implant was not better and his DGG dosage was not higher than in rabbits that learned. The failure to learn during DGG injections did not appear to be related to possible IO damage because this rabbit acquired CRs quickly during postacquisition training. However, this animal had been exposed to the lowest CS intensity among all of the subjects in this study because he was extremely sensitive to the vCS. We presume that this could explain his failure to acquire under DGG. Despite the low intensity of his vCS, this rabbit could be a borderline learner, having acquired some savings, given his rapid learning on the first day of postacquisition training, especially considering that this was preceded in all rabbits by a 40-trial extinction (retention) session.

The present study confirmed previous observations that, contrary to the cerebellar learning hypothesis, blocking glutamate neurotransmission in the IO abolishes previously learned CRs rather than inducing extinction. More importantly, we show that blocking IO task-related signals, at least enough to suppress CR expression, does not prevent vCS-evoked CR acquisition. With the cerebellum in an abnormal state of tonic activity, this suggests a remarkably resilient learning mechanism. Notwithstanding alternative explanations, the most parsimonious interpretation of our data is that glutamate-mediated IO signals are not needed for acquisition. Testing this proposition will require careful electrophysiological studies, such as recording US-evoked Purkinje cell complex spike activity in eyeblink microzones, to determine whether the DGG doses reported here are indeed capable of completely blocking all IO US signals. The general validity of this conclusion will also require testing whether $\mathrm{CR}$ acquisition to other CS modalities is similarly insensitive to blocking taskrelated IO signals.

\section{References}

Batini C, Billard JM, Daniel H (1985) Long term modification of cerebellar inhibition after inferior olive degeneration. Exp Brain Res 59:404-409. Medline

Bengtsson F, Jirenhed DA, Svensson P, Hesslow G (2007) Extinction of conditioned blink responses by cerebello-olivary pathway stimulation. Neuroreport 18:1479-1482. CrossRef Medline

Bracha V, Webster ML, Winters NK, Irwin KB, Bloedel JR (1994) Effects of muscimol inactivation of the cerebellar interposed-dentate nuclear complex on the performance of the nictitating membrane response in the rabbit. Exp Brain Res 100:453-468. CrossRef Medline

Bracha V, Irwin KB, Webster ML, Wunderlich DA, Stachowiak MK, Bloedel JR (1998) Microinjections of anisomycin into the intermediate cerebellum during learning affect the acquisition of classically conditioned responses in the rabbit. Brain Res 788:169-178. CrossRef Medline

Bracha V, Zbarska S, Parker K, Carrel A, Zenitsky G, Bloedel JR (2009) The cerebellum and eye-blink conditioning: learning versus network performance hypotheses. Neuroscience 162:787-796. CrossRef Medline

Carrel AJ, Zbarska S, Zenitsky GD, Bracha V (2012) A trigeminal conditioned stimulus yields fast acquisition of cerebellum-dependent conditioned eyeblinks. Behav Brain Res 226:189-196. CrossRef Medline

Colin F, Manil J, Desclin JC (1980) The olivocerebellar system I. Delayed and slow inhibitory effects: an overlooked salient feature of cerebellar climbing fibers. Brain Res 187:3-27. CrossRef Medline
De Zeeuw CI, Yeo CH (2005) Time and tide in cerebellar memory formation. Curr Opin Neurobiol 15:667-674. CrossRef Medline

Gellman R, Houk JC, Gibson AR (1983) Somatosensory properties of the inferior olive of the cat. J Comp Neurol 215:228 -243. CrossRef Medline

Halverson HE, Freeman JH (2010) Medial auditory thalamic input to the lateral pontine nuclei is necessary for auditory eyeblink conditioning. Neurobiol Learn Mem 93:92-98. CrossRef Medline

Jiménez-Díaz L, Navarro-Lopez Jde D, Gruart A, Delgado-Garcia JM (2004) Role of cerebellar interpositus nucleus in the genesis and control of reflex and conditioned eyelid responses. J Neurosci 24:9138-9145. CrossRef Medline

Jirenhed DA, Bengtsson F, Hesslow G (2007) Acquisition, extinction, and reacquisition of a cerebellar cortical memory trace. J Neurosci 27:2493-2502. CrossRef Medline

Kellett DO, Fukunaga I, Chen-Kubota E, Dean P, Yeo CH (2010) Memory consolidation in the cerebellar cortex. PLoS One 5:e11737. CrossRef Medline

Krupa DJ, Thompson JK, Thompson RF (1993) Localization of a memory trace in the mammalian brain. Science 260:989-991. CrossRef Medline

Lang EJ (2001) Organization of olivocerebellar activity in the absence of excitatory glutamatergic input. J Neurosci 21:1663-1675. Medline

McCormick DA, Steinmetz JE, Thompson RF (1985) Lesions of the inferior olivary complex cause extinction of the classically conditioned eyeblink response. Brain Res 359:120-130. CrossRef Medline

Medina JF, Nores WL, Mauk MD (2002) Inhibition of climbing fibres is a signal for the extinction of conditioned eyelid responses. Nature 416:330-333. CrossRef Medline

Mintz M, Lavond DG, Zhang AA, Yun Y, Thompson RF (1994) Unilateral inferior olive NMDA lesion leads to unilateral deficit in acquisition and retention of eyelid classical conditioning. Behav Neural Biol 61:218-224. CrossRef Medline

Montarolo PG, Palestini M, Strata P (1982) The inhibitory effect of the olivocerebellar input on the cerebellar Purkinje cells in the rat. J Physiol 332:187-202. Medline

Raymond JL, Lisberger SG, Mauk MD (1996) The cerebellum: a neuronal learning machine? Science 272:1126-1131. CrossRef Medline

Ryan SB, Detweiler KL, Holland KH, Hord MA, Bracha V (2006) A longrange, wide field-of-view infrared eyeblink detector. J Neurosci Methods 152:74-82. CrossRef Medline

Thompson RF (1986) The neurobiology of learning and memory. Science 233:941-947. CrossRef Medline

Van Ham JJ, Yeo CH (1992) Somatosensory trigeminal projections to the inferior olive, cerebellum and other precerebellar nuclei in rabbits. Eur J Neurosci 4:302-317. CrossRef Medline

Weiss C, Disterhoft JF, Gibson AR, Houk JC (1993) Receptive fields of single cells from the face zone of the cat rostral dorsal accessory olive. Brain Res 605:207-213. CrossRef Medline

Welsh JP, Harvey JA (1991) Pavlovian conditioning in the rabbit during inactivation of the interpositus nucleus. J Physiol 444:459-480. Medline

Welsh JP, Harvey JA (1998) Acute inactivation of the inferior olive blocks associative learning. Eur J Neurosci 10:3321-3332. CrossRef Medline

Yeo CH, Hardiman MJ, Glickstein M (1986) Classical conditioning of the nictitating membrane response of the rabbit. IV. Lesions of the inferior olive. Exp Brain Res 63:81-92. Medline

Zbarska S, Bracha V (2012) Assessing the role of inferior olivary sensory signaling in the expression of conditioned eyeblinks using a combined glutamate/ GABAA receptor antagonist protocol. J Neurophysiol 107:273-282. CrossRef Medline

Zbarska S, Holland EA, Bloedel JR, Bracha V (2007) Inferior olivary inactivation abolishes conditioned eyeblinks: extinction or cerebellar malfunction? Behav Brain Res 178:128-138. CrossRef Medline

Zbarska S, Bloedel JR, Bracha V (2008) Cerebellar dysfunction explains the extinction-like abolition of conditioned eyeblinks after NBQX injections in the inferior olive. J Neurosci 28:10-20. CrossRef Medline 